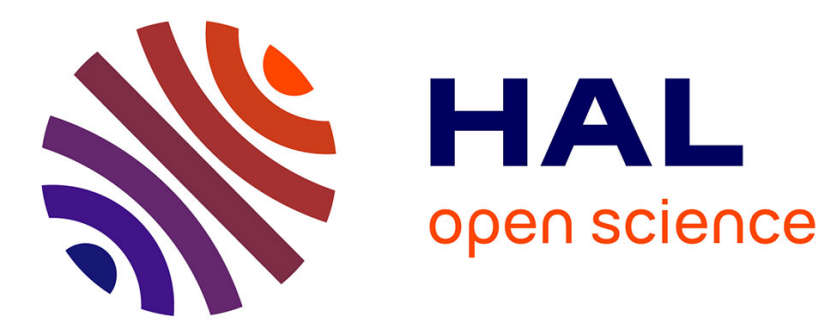

\title{
Weighted enumeration of spanning subgraphs in locally tree-like graphs \\ Justin Salez
}

\section{To cite this version:}

Justin Salez. Weighted enumeration of spanning subgraphs in locally tree-like graphs. Random Structures and Algorithms, 2013, 43 (3), pp.377-397. 10.1002/rsa.20436 . inria-00577234

\section{HAL Id: inria-00577234 \\ https://hal.inria.fr/inria-00577234}

Submitted on 16 Mar 2011

HAL is a multi-disciplinary open access archive for the deposit and dissemination of scientific research documents, whether they are published or not. The documents may come from teaching and research institutions in France or abroad, or from public or private research centers.
L'archive ouverte pluridisciplinaire HAL, est destinée au dépôt et à la diffusion de documents scientifiques de niveau recherche, publiés ou non, émanant des établissements d'enseignement et de recherche français ou étrangers, des laboratoires publics ou privés. 


\title{
The cavity method for counting spanning subgraphs subject to local constraints
}

\author{
Justin Salez*
}

March 16, 2011

\begin{abstract}
Using the theory of negative association for measures and the notion of random weak limits of sparse graphs, we establish the validity of the cavity method for counting spanning subgraphs subject to local constraints in asymptotically tree-like graphs. Specifically, the corresponding free entropy density is shown to converge along any sequence of graphs whose random weak limit is a tree, and the limit is directly expressed in terms of the unique solution to a limiting cavity equation. On a Galton-Watson tree, the latter simplifies into a recursive distributional equation which can be solved explicitely. As an illustration, we provide an explicit-limit formula for the $b$-matching number of an Erdős-Rényi random graph with fixed average degree and diverging size, for any $b \in \mathbb{N}$.
\end{abstract}

\section{Introduction}

The general framework we consider is that of a finite graph $G=(V, E)$, in which spanning subgraphs are weighted according to their local aspect around each vertex as follows :

$$
\mu(F)=\prod_{i \in V} \mu_{i}\left(F \cap E_{i}\right)
$$

Here, a spanning subgraph $(V, F)$ is identified with its egde-set $F \subseteq E$, and each $\mu_{i}$ is a given non-negative function over the subsets of $E_{i}:=\{e \in E$; $e$ is incident to $i\}$. We call $\mu$ the global measure induced by the local measures $\mu_{i}, i \in V$. Of particular interest in combinatorial optimization is the number

$$
M(G)=\max \{|F|: F \in \operatorname{supp}(\mu)\},
$$

which is the maximum possible size of a spanning subgraph $F$ satisfying the local contraint $\mu_{i}\left(F \cap E_{i}\right)>0$ at every node $i \in V$. More generally, counting the weighted number of spanning subgraphs of each given size in $G$, i.e. determining the generating polynomial

$$
Z(G ; t)=\sum_{F \subseteq E} \mu(F) t^{|F|}
$$

\footnotetext{
*INRIA-École Normale Supérieure - France. Email: justin.salez@ens.fr
} 
is a fundamental task, of which many combinatorial problems are special instances. Intimately related to this is the study of a random spanning subgraph $\mathcal{F}$ sampled from the Gibbs-Boltzmann law :

$$
\mathbb{P}_{G}^{t}(\mathcal{F}=F)=\frac{\mu(F) t^{|F|}}{Z(G ; t)},
$$

where $t>0$ is a variable parameter called the activity. In particular, the expected size of $\mathcal{F}$ is called the energy $U(G ; t)$ and is connected to $Z(G ; t)$ via the elementary identity

$$
U(G ; t)=t \frac{d}{d t} \log Z(G ; t) .
$$

Our concern is the behavior of these quantities in the infinite volume limit : $|V| \rightarrow \infty,|E|=\Theta(|V|)$.

Originating from spin glass theory [26, the cavity method is a powerful nonrigorous technique for evaluating such asymptotics on graphs that are locally tree-like. Essentially, the heuristic consists in neglecting cycles in order to obtain an approximate local fixed point equation for the marginals of the Gibbs-Boltzmann law. Despite its remarkable practical efficiency and the mathematical confirmation of its analytical predictions for various important models [31, 1, 20, 28, 1, 16, 13], this ansatz is still far from being completely understood, and the exact conditions for its validity remain unknown. More precisely, two crucial questions arise in presence of cycles :

1. convergence : is there a unique, globally attractive fixed point to the cavity equation?

2. correctness : if yes, does it have any relation to the Gibbs-Boltzmann marginals?

In this paper, we exhibit a general condition under which the cavity method is valid for counting spanning subgraphs subject to local constaints. Specifically, we positively answer question 1 for arbitrary finite graphs (Theorem 2), under the only assumption that each local measure enjoys a certain form of negative association which we call the cavity-monotone property, and which simply boils down to ultra-logconcavity in the exchangeable case. Regarding question 2, we use the framework of local weak convergence [8, 4] and the notion of unimodularity [3] to prove asymptotical correctness for any sequence of graphs whose random weak limit is concentrated on trees (Theorem 4 ). This includes many classical sequences of diluted graphs, such as random $d$-regular graphs, Erdős-Rényi random graphs with fixed average degree, or more generally random graphs with a prescribed degree distribution. In all these examples, the limit is a unimodular Galton-Watson ( $U G W$ ) tree. Thanks to the distributional self-similarity of such a tree, the cavity equation simplifies into a recursive distributional equation which may be solved explicitely. As a motivation, let us first describe the implications of our work in the special case of b-matchings.

A famous combinatorial structure that fits in the above framework is obtained by fixing $b \in \mathbb{N}$ and taking $\mu_{i}(F)=\mathbf{1}(|F| \leq b)$ for all $i \in V$ : the induced global measure $\mu$ is then nothing but the counting measure for $b$-matchings in $G$, i.e. spanning subgraphs with maximum degree at most $b$. The reader is refered to the monograph [30] for a complete survey on $b$-matchings, and to [24] for the important case of matchings $(b=1)$. The associated quantities $M_{b}(G)$ and $Z_{b}(G ; t)$ are important graph invariants respectively known as the $b$-matching number and $b$-matching polynomial. Determining $Z_{1}(G ; t)$ is a classical example of a computationally hard problem [32], although efficient approximation algorithms have been designed [6, 5]. The mathematical properties of $Z_{b}(G ; t)$ have been investigated in detail, notably in the case $b=1$ for the purpose of understanding monomer-dimer systems [21, 10]. Interestingly, the geometry of the complex zeros of $Z_{b}(G, t)$ has been proven to be quite remarkable (see [21] for $b=1$, [29] for $b=2$, and 34] for the general case). Regarding $M_{1}(G)$, the first results in the infinite volume 
limit were obtained by Karp and Sipser [23] for the Erdős-Rényi random graph $G_{n}$ with average degree $c>0$ on $n$ vertices:

$$
\frac{1}{n} M_{1}\left(G_{n}\right) \underset{n \rightarrow \infty}{\stackrel{P}{\longrightarrow}} 1-\frac{t_{c}+e^{-c t_{c}}+c t_{c} e^{-c t_{c}}}{2},
$$

where $t_{c} \in(0,1)$ is the smallest root of $t=e^{-c e^{-c t}}$. The analysis has then been extended to random graphs with a log-concave degree profile [12], and finally to any graph sequence that converges in the local weak sense 13]. Contrastingly, only little is known for $b \geq 2$ : to the best of our knowledge, the limit of $\frac{1}{\left|V_{n}\right|} M_{b}\left(G_{n}\right)$ is only known to exist in the Erdős-Rényi case [19], and could not be explicitely determined. As a special case of our main result, it will follow that

Theorem 1 (b-matchings in locally tree-like graphs) For any sequence of finite graphs $\left(G_{n}\right)_{n \in \mathbb{N}}$ satisfying $\left|E_{n}\right|=O\left(\left|V_{n}\right|\right)$ and whose random weak limit $\mathcal{L}$ is concentrated on trees, the limits

$$
f_{b}(\mathcal{L}):=\lim _{n \rightarrow \infty} \frac{1}{\left|V_{n}\right|} \log Z_{b}\left(G_{n} ; t\right) \quad \text { and } \quad m_{b}(\mathcal{L}):=\lim _{n \rightarrow \infty} \frac{M_{b}\left(G_{n}\right)}{\left|V_{n}\right|}
$$

exist and depend only on the random weak limit $\mathcal{L}$. When $\mathcal{L}$ is a $U G W$ tree, we have the explicit formula

$$
m_{b}(\mathcal{L}):=\min _{s \in[0,1]}\left\{b-\frac{b}{2} g_{b}(s)-\frac{b}{2}\left(g_{b} \circ f_{b}\right)(s)+\frac{c}{2} f_{b}(s)\left(f_{b} \circ f_{b}\right)(s)\right\},
$$

where $c, f, g$ are defined in terms of the degree generating function $\phi(s)=\sum_{k} \pi_{k} s^{k}$ as follows :

$$
c=\phi^{\prime}(1), \quad f_{b}(s)=\frac{1}{c} \sum_{k=0}^{b-1} \frac{s^{k} \phi^{(k+1)}(1-s)}{k !} \quad \text { and } \quad g_{b}(s)=\sum_{k=0}^{b} \frac{s^{k} \phi^{(k)}(1-s)}{k !} .
$$

Moreover, any $s$ where the above minimum is achieved must be a root of $s=\left(f_{b} \circ f_{b}\right)(s)$.

For example, in the case of Erdős-Rényi random graphs with average degree $c>0$ on $n$ vertices, the random weak limit $\mathcal{L}$ is a.s. the law of a UGW tree with Poisson(c) degree distribution, and hence,

$$
\frac{1}{n} M_{b}\left(G_{n}\right) \underset{n \rightarrow \infty}{\stackrel{a . s .}{\longrightarrow}} \min _{s \in[0,1]}\left\{b-\frac{b}{2} \varphi_{b+1}(s)-\frac{b}{2}\left(\varphi_{b+1} \circ \varphi_{b}\right)(s)+\frac{c}{2} \varphi_{b}(s)\left(\varphi_{b} \circ \varphi_{b}\right)(s)\right\},
$$

where we have set

$$
\varphi_{b}(s)=e^{-c t} \sum_{k=0}^{b-1} \frac{c s^{k}}{k !} .
$$

Since any $s$ where the minimum is achieved must satisfy $s=\left(\varphi_{b} \circ \varphi_{b}\right)(s)$, we recover exactly (6) in the special case of matchings $(b=1)$.

The paper is organized as follows : in section 2, we recall the basic notions and properties pertaining to measures over subsets, which will be of constant use throughout the paper. In section 3, we define and study the cavity equation associated to a finite network. In section 4 , we extend the results to infinite networks that arise as local weak limits of finite networks. Finally, section 5 is devoted to the study of the cavity equation in the limit of infinite activity, and to its explicit resolution in the case of $b$-matchings. 


\section{Preliminaries}

In this section, we define the important notions pertaining to (non-negative) measures $\mu$ over the subsets of an arbitrary finite ground set $E$. Later on, these will be specialized to the local measures $\left(\mu_{i}\right)_{i \in V}$ attached to the vertices of a graph $G$. First, $\mu$ is caracterized by its multivariate generating polynomial

$$
Z(\mathbf{w})=\sum_{F \subseteq E} \mu(F) \mathbf{w}^{F}
$$

where $\mathbf{w}=\left(w_{e}\right)_{e \in E}$ and $\mathbf{w}^{F}=\prod_{e \in F} w_{e}$. Since $Z$ is affine in each $w_{e}, e \in E$, it can be decomposed as

$$
Z(\mathbf{w})=w_{e} Z^{/ e}\left(\mathbf{w}^{\prime}\right)+Z^{\backslash e}\left(\mathbf{w}^{\prime}\right),
$$

where $\mathbf{w}^{\prime}=\left(w_{f}\right)_{f \neq e}$ and $Z^{\backslash e}, Z^{/ e}$ are the multi-affine polynomials with ground set $E \backslash e$ respectively obtained from $Z$ by setting the variable $w_{e}$ to 0 (deletion) and differentiating with respect to $w_{e}$ (contraction). By definition, the cavity ratio of the pair $(\mu, e)$ is then simply the multi-affine rational function

$$
\Gamma_{\mu}^{e}\left(\mathbf{w}^{\prime}\right)=\frac{Z^{/ e}\left(\mathbf{w}^{\prime}\right)}{Z^{\backslash e}\left(\mathbf{w}^{\prime}\right)}
$$

When positive values are assigned to the variables (a so-called external field), we may consider the probability distribution $\mathbb{P}_{\mu}^{\mathbf{w}}(\mathcal{F}=F)=\mu(F) \mathbf{w}^{F} / Z(\mathbf{w})$. A quantity of interest is the expected size of $\mathcal{F}$ when viewed as a function of the external field. We call this the energy :

$$
U_{\mu}(\mathbf{w})=\mathbb{E}_{\mu}^{\mathbf{w}}[|\mathcal{F}|]
$$

From the decomposition (8), it follows immediately that

$$
\mathbb{P}_{\mu}^{\mathbf{w}}(e \in \mathcal{F})=\frac{w_{e} \Gamma_{\mu}^{e}\left(\mathbf{w}^{\prime}\right)}{1+w_{e} \Gamma_{\mu}^{e}\left(\mathbf{w}^{\prime}\right)}, \quad \text { hence } \quad U_{\mu}(\mathbf{w})=\sum_{e \in E} \frac{w_{e} \Gamma_{\mu}^{e}\left(\mathbf{w}^{\prime}\right)}{1+w_{e} \Gamma_{\mu}^{e}\left(\mathbf{w}^{\prime}\right)}
$$

Note that the supremum of the energy is exactly the $\operatorname{rank}$ of $\mu: \operatorname{rank}(\mu)=\max \{|\mathrm{F}| ; \mathrm{F} \in \operatorname{supp}(\mu)\}$. The following properties will be of crucial importance throughout the paper.

Definition 1 (Cavity-monotone measures) The measure $\mu$ is called

- Rayleigh if every two distinct ground elements $e \neq f$ are negatively correlated in $\mathcal{F}$ :

$$
\forall \mathbf{w}>0, \mathbb{P}_{\mu}^{\mathbf{w}}(e \in \mathcal{F}, f \in \mathcal{F}) \leq \mathbb{P}_{\mu}^{\mathbf{w}}(e \in \mathcal{F}) \mathbb{P}_{\mu}^{\mathbf{w}}(f \in \mathcal{F})
$$

- Size-increasing if every ground element e positively influences the total size $|\mathcal{F}|$ :

$$
\forall \mathbf{w}>0, \mathbb{E}_{\mu}^{\mathbf{w}}\left[|\mathcal{F}| \mathbf{1}_{(e \in \mathcal{F})}\right]>\mathbb{E}_{\mu}^{\mathbf{w}}[|\mathcal{F}|] \mathbb{P}_{\mu}^{\mathbf{w}}(e \in \mathcal{F})
$$

- Cavity-monotone if its satisfies $\mu(\emptyset)>0$ and the two above properties.

Rayleigh measures were introduced in the context of matroid theory [33 the modern theory of negative dependence for probability measures [27, 22]. Cavity-monotone measures will play a major role in our study, for the following elementary reason. 


\section{Lemma 1 (Monotony of energy and cavity ratios)}

$$
\begin{aligned}
\mu(\emptyset)>0 & \Longleftrightarrow \text { the cavity ratios } \Gamma_{\mu}^{e}, e \in E \text { are well-defined on }[0, \infty) \times \ldots \times[0, \infty) . \\
\mu \text { is Rayleigh } & \Longleftrightarrow \text { the cavity ratios } \Gamma_{\mu}^{e}, e \in E \text { are non-increasing in each variable. } \\
\mu \text { is size-increasing } & \Longleftrightarrow \text { the energy } U_{\mu} \text { is increasing in each variable; } \\
& \Longleftrightarrow \text { for each } e \in E \text { and } \mathbf{w}>0, t \mapsto t \Gamma_{\mu}^{e}\left(t \mathbf{w}^{\prime}\right) \text { is increasing. }
\end{aligned}
$$

Proof. Differentiating the corresponding quantities and playing with the definition of $\mathbb{P}_{\mu}^{\mathbf{w}}$ easily yields

$$
\begin{aligned}
\frac{\partial \Gamma_{\mu}^{e}\left(\mathbf{w}^{\prime}\right)}{\partial w_{f}} & =\left(\mathbb{P}_{\mu}^{\mathbf{w}}(e \in \mathcal{F}, f \in \mathcal{F})-\mathbb{P}_{\mu}^{\mathbf{w}}(e \in \mathcal{F}) \mathbb{P}_{\mu}^{\mathbf{w}}(f \in \mathcal{F})\right) / w_{e} w_{f} \mathbb{P}_{\mu}^{\mathbf{w}}(e \notin \mathcal{F})^{2} . \\
\frac{\partial U_{\mu}(\mathbf{w})}{\partial w_{e}} & =\left(\mathbb{E}_{\mu}^{\mathbf{w}}\left[|\mathcal{F}| \mathbf{1}_{(e \in \mathcal{F})}\right]-\mathbb{E}_{\mu}^{\mathbf{w}}[|\mathcal{F}|] \mathbb{P}_{\mu}^{\mathbf{w}}(e \in \mathcal{F})\right) / w_{e} . \\
\frac{\partial t \Gamma_{\mu}^{e}\left(t \mathbf{w}^{\prime}\right)}{\partial t} & =\left(\mathbb{E}_{\mu}^{\mathbf{w}}\left[|\mathcal{F}| \mathbf{1}_{(e \in \mathcal{F})}\right]-\mathbb{E}_{\mu}^{\mathbf{w}}[|\mathcal{F}|] \mathbb{P}_{\mu}^{\mathbf{w}}(e \in \mathcal{F})\right) / t w_{e} \mathbb{P}_{\mu}^{\mathbf{w}}(e \notin \mathcal{F})^{2} .
\end{aligned}
$$

Remark 1 (Matroids) Interestingly, the support of a cavity-monotone measure admits a remarkable structure : it follows from [3, Theorem 4.6] that for $\mu$ Rayleigh with $\mu(\emptyset)>0, I=\operatorname{supp} \mu$ is a matroid:

- I is not empty ;

- If $B \in I$ and $A \subseteq B$, then $A \in I$;

- If $A, B \in I$ and $|A|<|B|$, then $\exists e \in B \backslash A$ such that $A \cup e \in I$.

The cavity-monotone property admits a particularly simple caracterization in the important case where $\mu$ is exchangeable, i.e. $\mu(F)=c(|F|)$ for some non-negative coefficients $c(0), \ldots, c(m), m=|E|$ :

Lemma 2 (The exchangeable case) An exchangeable measure $\mu$ is cavity-monotone if and only if

1. $c$ is log-concave, i.e. $c^{2}(k) \geq c(k-1) c(k+1)$ for all $0<k<m$, and

2. the support $\{0 \leq k \leq m: c(k)>0\}$ is an interval containing 0 and 1 .

In particular, so is the measure $\mu(F)=\mathbf{1}_{(|F| \leq b)}$ describing the local constraints of a $b$-matching.

Proof of Lemma 国. The result essentially follows from the work of Pemantle [27]. Indeed, Theorem 2.7 therein guarantees that $\mu$ is Rayleigh if and only if the sequence $c$ is $\log$-concave and its support is an interval. That the latter must contain 0 is nothing but the last property in the definition of a cavity-monotone measure. That it is not reduced to 0 is imposed by the strict inequality in the sizeincreasing property. Conversely, let us show that any exchangeable measure $\mu$ with $c(0)>0$ and $c(1)>0$ is indeed size-increasing. Fix an external field $\mathbf{w}>0$. By Lemma 2.9 in [27], the law obtained from $\mathbb{P}_{\mu}^{\mathbf{w}}$ by conditionning on the event $\{|\mathcal{F}|=k\}$ is stochastically increasing in $k$. By Proposition 1.2 in [27, this implies in particular that for every $e \in E$, the following weak inequality holds :

$$
\mathbb{E}_{\mu}^{\mathbf{w}}[|\mathcal{F}| \mid e \in \mathcal{F}] \geq \mathbb{E}_{\mu}^{\mathbf{w}}[|\mathcal{F}|] .
$$

Note that the condition $c(1)>0$ guarantees that this conditional expectation is well-defined. Since we have not yet used the fact that $c(0)>0$, the above inequality remains true if one changes the coefficient $c(0)$ to 0 . Setting it then back to its initial (positive) value does not affect the left-hand side, but strictly decreases the right-hand side, hence the desired strict inequality. 


\section{The cavity equation on finite networks}

Let $G=(V, E)$ be a finite graph at the vertices of which some local measures $\mu_{i}, i \in V$ are specified. We call the resulting object a network. A configuration $\mathbf{x}$ is an assignment of numbers $x_{i \rightarrow j} \geq 0$ to every oriented edge $i \rightarrow j \in \vec{E}$. Starting from a configuration $\mathbf{x}$, we define a new configuration $\mathbf{y}=\Gamma_{G}(\mathbf{x})$ by

$$
y_{i \rightarrow j}=\Gamma_{\mu_{i}}^{i j}\left(x_{k \rightarrow i}: k \in \partial i \backslash j\right),
$$

where $\partial i$ denotes the set of all neighbors of $i$. Each $x_{i \rightarrow j}$ may be thought of as a message sent by $i$ to $j$ along the edge $i j$, and $\Gamma_{G}$ as a local rule for propagating messages. For $t>0$, the fixed point equation

$$
\mathbf{x}=t \Gamma_{G}(\mathbf{x})
$$

is called the cavity equation at activity $t$ on the network $G$. Its relation to the global measure $\mu$ induced by the $\left(\mu_{i}\right)_{i \in V}$ is revealed by the following well-known result.

Lemma 3 (Validity on trees) Assume that $G$ is finite and acyclic. Then, for every activity $t>0$,

1. convergence : the cavity equation admits a unique solution $\mathbf{x}(t)$, which can be reached from any initial configuration by iterating $t \Gamma_{G}$ a number of times equal to the diameter of $G$;

2. correctness : for every $i \in V$, the exact marginal law of $\mathcal{F} \cap E_{i}$ under the Gibbs-Boltzmann law $\mathbb{P}_{G}^{t}$ is given by directly imposing the external field $\left\{x_{j \rightarrow i}(t): j \in \partial i\right\}$ onto the local measure $\mu_{i}$.

The important consequence is that on trees, the energy $U(G ; t)$ can be determined using only local operations :

$$
U(G ; t)=\frac{1}{2} \sum_{i \in V} U_{\mu_{i}}\left(x_{j \rightarrow i}(t): j \in \partial i\right)=\sum_{i j \in E} \frac{x_{j \rightarrow i}(t) x_{i \rightarrow j}(t)}{t+x_{j \rightarrow i}(t) x_{i \rightarrow j}(t)}
$$

where the second equality is obtained by applying (11) to each $\mu_{i}, i \in V$.

Proof of Lemma 3. When $i$ is a leaf, the message $y_{i \rightarrow j}$ defined by equation (12) does not depend at all on the initial configuration $\mathbf{x}$. Iterating this argument immediately proves the convergence part, and we now focus on correctness. Let $G=(V, E)$ be a finite tree, o a vertex, and $i$ a neighbour of o. We let $G_{i \rightarrow \circ}$ denote the subtree induced by $\circ$ and all vertices that the edge $i \circ$ separates from $\circ$. Now assume that $G$ is equipped with local measures, and let $G_{i \rightarrow \circ}$ inherit from these local measures, except for $\mu_{\circ}$ which we replace by the trivial local measure with constant value 1 . With these notations, any spanning subgraph $F \subseteq E$ can be uniquely decomposed as the disjoint union of a subset $I \subseteq E_{\circ}$ and a spanning subgraph $F_{i}$ on each $G_{i \rightarrow \circ}, i \in \partial \circ$, with $i \circ \notin F_{i}$. Thus, writing $\mu_{G}$ for the global measure on the network $G$, we have for any $t>0$,

$$
\mu_{G}(F) t^{|F|}=\mu_{\circ}(I) \prod_{i \in I} t^{\left|F_{i}\right|+1} \mu_{G_{i \rightarrow \circ}}\left(F_{i} \cup i \circ\right) \prod_{i \notin I} t^{\left|F_{i}\right|} \mu_{G_{i \rightarrow 0}}\left(F_{i}\right) .
$$

Fixing $I$ and summing over all possible values for $F_{i}, i \in \partial \circ$, we obtain

$$
\begin{aligned}
\mathbb{P}_{G}^{t}\left(\mathcal{F} \cap E_{\circ}=I\right) & =C \mu_{\circ}(I) \prod_{i \in I} \mathbb{P}_{G_{i \rightarrow \circ}}^{t}(i \circ \in \mathcal{F}) \prod_{i \notin I} \mathbb{P}_{G_{i \rightarrow \circ}}^{t}(i \circ \notin \mathcal{F}) . \\
& =C^{\prime} \mu_{\circ}(I) \prod_{i \in I} \frac{\mathbb{P}_{G_{i \rightarrow \circ}}^{t}(i \circ \in \mathcal{F})}{\mathbb{P}_{G_{i \rightarrow \circ}}^{t}(i \circ \notin \mathcal{F})}
\end{aligned}
$$


where $C, C^{\prime}$ are normalizing constants that do not depend on $I$. This already proves that the law of $\mathcal{F} \cap E_{\circ}$ can be obtained from the local measure $\mu_{\circ}$ by imposing on each edge $i \circ \in E_{\circ}$ the external field

$$
x_{i \rightarrow 0}(t):=\frac{\mathbb{P}_{G_{i \rightarrow 0}}^{t}(i \circ \in \mathcal{F})}{\mathbb{P}_{G_{i \rightarrow 0}}^{t}(i \circ \notin \mathcal{F})} .
$$

In turn, this ratio can now be computed by applying the result to the vertex $i$ in the network $G_{i \rightarrow 0}$ :

$$
\frac{\mathbb{P}_{G_{i \rightarrow 0}}^{t}(i \circ \in \mathcal{F})}{\mathbb{P}_{G_{i \rightarrow 0}}^{t}(i \circ \notin \mathcal{F})}=t \Gamma_{\mu_{i}}^{i \circ}\left(x_{k \rightarrow i}(t): k \in \partial i \backslash \circ\right),
$$

which shows that the configuration $\mathbf{x}(t)$ defined on $G$ by (15) satisfies the cavity equation (13).

There are two distinct parts in Lemma 3 : convergence and correctness. As we will now show, the former extends to arbitrary graphs under the only assumption that each local measure is cavity-monotone. Henceforth, such a network will be called a cavity-monotone network.

Theorem 2 (Convergence on finite cavity-monotone networks) On a finite cavity-monotone network, the cavity equation admits a unique, globally attractive fixed point $\mathbf{x}(t)$ at any activity $t>0$.

Proof. Fixing $t>0$ and starting with the minimal configuration $\mathbf{x}^{0}:=\mathbf{0}$, we set inductively

$$
\mathbf{x}^{k+1}(t):=t \Gamma_{G}\left(\mathbf{x}^{k}(t)\right),
$$

for all $k \in \mathbb{N}$. By Lemma 1 , the Rayleigh property of the local measures $\mu_{i}, i \in V$ ensures that $\Gamma_{G}$ is coordinate-wise non-increasing on the space of configurations. Therefore, the limiting configuration

$$
\mathbf{x}^{-}(t):=\lim _{k \rightarrow \infty} \uparrow \mathbf{x}^{2 k}(t) \quad \text { and } \quad \mathbf{x}^{+}(t):=\lim _{k \rightarrow \infty} \downarrow \mathbf{x}^{2 k+1}(t)
$$

exist, and any fixed point $\mathbf{x}=t \Gamma_{G}(\mathbf{x})$ must satisfy $\mathbf{x}^{-}(t) \leq \mathbf{x} \leq \mathbf{x}^{+}(t)$. Moreover, $\Gamma_{G}$ is clearly continuous with respect to the product topology on configurations, so that $t \Gamma_{G}\left(\mathbf{x}^{-}(t)\right)=\mathbf{x}^{+}(t)$ and $t \Gamma_{G}\left(\mathbf{x}^{+}(t)\right)=$ $\mathbf{x}^{-}(t)$. Thus, the existence of unique globally attractive solution to (13) boils down to the equality

$$
\mathbf{x}^{-}(t)=\mathbf{x}^{+}(t)
$$

Now applying (11) to the local measure at a fixed vertex $i \in V$ yields

$$
U_{\mu_{i}}\left(x_{j \rightarrow i}^{-}(t): j \in \partial i\right)=\sum_{j \in \partial i} \frac{x_{j \rightarrow i}^{-}(t) x_{i \rightarrow j}^{+}(t)}{t+x_{j \rightarrow i}^{-}(t) x_{i \rightarrow j}^{+}(t)}, \quad U_{\mu_{i}}\left(x_{j \rightarrow i}^{+}(t): j \in \partial i\right)=\sum_{j \in \partial i} \frac{x_{j \rightarrow i}^{+}(t) x_{i \rightarrow j}^{-}(t)}{t+x_{j \rightarrow i}^{+}(t) x_{i \rightarrow j}^{-}(t)} .
$$

Summing over all vertices $i \in V$, we therefore obtain

$$
\begin{aligned}
\sum_{i \in V} U_{\mu_{i}}\left(x_{j \rightarrow i}^{-}(t): j \in \partial i\right) & =\sum_{i j \in E}\left(\frac{x_{j \rightarrow i}^{-}(t) x_{i \rightarrow j}^{+}(t)}{t+x_{j \rightarrow i}^{-}(t) x_{i \rightarrow j}^{+}(t)}+\frac{x_{j \rightarrow i}^{+} x_{i \rightarrow j}^{-}(t)}{t+x_{j \rightarrow i}^{+}(t) x_{i \rightarrow j}^{-}(t)}\right) \\
& =\sum_{i \in V} U_{\mu_{i}}\left(x_{j \rightarrow i}^{+}(t): j \in \partial i\right) .
\end{aligned}
$$

This implies (17), since by Lemma 1 each $U_{\mu_{i}}, i \in V$ is strictly increasing in every coordinate. 


\section{The limit of infinite volume}

In the previous section, we have established existence and uniqueness of a cavity solution on any finite cavity-monotone network. Our concern now is its asymptotical meaning as the size of the underlying graph tends to infinity. Following the principles of the objective method [4], we will replace the asymptotical analysis of our finite networks by the direct study of their infinite limits.

\subsection{Random weak limits}

We first briefly recall the framework of local convergence, introduced by Benjamini and Schramm 80 and developped further by Aldous and Steele [4]. Examples of successful uses include [3, 9, 18, 25, 15, 14, 13, 16. Here, a network will be simply a denumerable graph $G=(V, E)$ whose vertices are equipped with local measures $\mu_{i}, i \in V$. A rooted network $(G, \circ)$ is a network together with the specification of a particular vertex $\circ \in V$, called the root. For $\varepsilon \geq 0$, we write $\left(G^{\prime}, \circ^{\prime}\right) \stackrel{\varepsilon}{\equiv}(G, \circ)$ if there exists a bijection $\gamma: V \rightarrow V^{\prime}$ that preserves

- the root : $\gamma(0)=\circ^{\prime}$;

- the adjacency : $i j \in E \Longleftrightarrow \gamma(i) \gamma(j) \in E^{\prime}$;

- the support of the local measures : $\mu_{i}(F)>0 \Longleftrightarrow \mu_{\gamma(i)}^{\prime}(\gamma(F))>0$, with $\gamma(F)=\{\gamma(i) \gamma(j): i j \in F\}$.

- the values of the local measures, up to $\varepsilon:\left|\mu_{\gamma(i)}^{\prime}(\gamma(F))-\mu_{i}(F)\right| \leq \varepsilon$.

We let $\mathcal{G}_{*}$ denote the set of all locally finite connected rooted networks considered up to the isomorphism relation $\stackrel{0}{\equiv}$. In the space $\mathcal{G}_{*}$, a sequence $\left\{\left(G_{n}, \circ_{n}\right) ; n \in \mathbb{N}\right\}$ converges locally to $(G, \circ)$ if for every radius $k \in \mathbb{N}$ and every $\varepsilon>0$, there is $n_{k, \varepsilon} \in \mathbb{N}$ such that

$$
n \geq n_{k} \Longrightarrow\left[G_{n}, \circ_{n}\right]_{k} \stackrel{\varepsilon}{=}[G, \circ]_{k},
$$

where $[G, \circ]_{k}$ denotes the finite rooted network obtained by keeping only the vertices lying at graphdistance at most $k$ from $\circ$. It is not hard to construct a distance which metrizes this notion of convergence and turns $\mathcal{G}$ into a complete separable metric space. We can thus import the usual machinery of weak convergence of probability measures on Polish spaces (see e.g. [11).

Uniform rooting is a natural procedure for turning a finite deterministic network $G$ into a random element of $\mathcal{G}_{*}$ : one simply chooses uniformly at random a vertex ○ to be the root, and restrains $G$ to the connected component of $\circ$. If $\left(G_{n}\right)_{n \in \mathbb{N}}$ is a sequence of finite networks and if the sequence of their laws under uniform rooting admits a weak limit $\mathcal{L} \in \mathcal{P}\left(\mathcal{G}_{*}\right)$, we call $\mathcal{L}$ the random weak limit of the sequence $\left(G_{n}\right)_{n \in \mathbb{N}}$. In [3], it was shown that any such limit enjoys a remarkable invariance property known as unimodularity : let $\mathcal{G}_{* *}$ denote the space of locally finite connected networks with an ordered pair of distinguished adjacent vertices $(G, \circ, i)$, taken up to the natural isomorphism relation and endowed with the natural topology. A measure $\mathcal{L} \in \mathcal{P}\left(\mathcal{G}_{*}\right)$ is called unimodular if it satisfies the Mass-Transport Principle : for any Borel function $f: \mathcal{G}_{* *} \rightarrow[0, \infty]$,

$$
\mathcal{L}\left[\sum_{i \in \partial \circ} f(G, \circ, i)\right]=\mathcal{L}\left[\sum_{i \in \partial \circ} f(G, i, \circ)\right],
$$

where we have written $\mathcal{L}[\cdot]$ for the expectation with respect to $\mathcal{L}$. This is a deep and powerful notion, which we will now use to extend the results of section 3 to the infinite setting. 


\subsection{Main result : validity of the cavity method on unimodular trees}

The definition of $\Gamma_{G}$ remains valid for any locally finite network $G$. When the latter is cavity-monotone, the configurations $\mathbf{x}^{-}(t), \mathbf{x}^{+}(t)$ introduced in the proof of Theorem 2 remain perfectly well-defined, and the convergence of the cavity method again boils down to the identity $\mathbf{x}^{-}(t)=\mathbf{x}^{+}(t)$. However, the proof of the latter involves a summation over all edges, which is no longer valid in the infinite setting. Instead, the desired $\mathbf{x}^{-}(t)=\mathbf{x}^{+}(t)$ will be derived from unimodularity, and will thus hold for any random weak limit of finite networks. Indeed, applying the Mass-Transport Principle to the function

$$
f(G, \circ, i):=\frac{x_{i \rightarrow \circ}^{-}(t) x_{\circ \rightarrow i}^{+}(t)}{t+x_{i \rightarrow \circ}^{-}(t) x_{\circ \rightarrow i}^{+}(t)}
$$

yields $\mathcal{L}\left[U_{\mu_{\circ}}\left(x_{i \rightarrow \circ}^{-}(t): i \in \partial \circ\right)\right]=\mathcal{L}\left[U_{\mu_{\circ}}\left(x_{i \rightarrow \circ}^{+}(t): i \in \partial \circ\right)\right](f$ is Borel as the pointwise limit of continuous functions). Under the assumption $\mathcal{L}\left[\operatorname{rank}\left(\mu_{\circ}\right)\right]<\infty$, this expectation is finite, and the size-increasing property of $\mu_{\circ}$ then implies that $\mathcal{L}$-almost surely, $x_{i \rightarrow 0}^{-}(t)=x_{i \rightarrow 0}^{+}(t)$ for all $i \in \partial \circ$. This automatically extends to every oriented edge since under unimodularity, everything shows up at the root (another fruitful application of the Mass-Transport-Principle, see [3, Lemma 2.3]). We state this as a Theorem.

Theorem 3 (Convergence of the cavity method on unimodular networks) Let $\mathcal{L}$ be a unimodular probability measure supported by cavity-monotone networks. If $\mathcal{L}\left[\operatorname{rank}\left(\mu_{\circ}\right)\right]<\infty$, then the cavity equation admits $\mathcal{L}-$ a.-s. a unique, globally attractive solution $\mathbf{x}(t)$ at any activity $t>0$.

By analogy with formula (14) in the finite case, the (now well-defined) quantity

$$
u(\mathcal{L} ; t)=\frac{1}{2} \mathcal{L}\left[\sum_{i \in \partial \circ} \frac{x_{i \rightarrow \circ}(t) x_{\circ \rightarrow i}(t)}{t+x_{i \rightarrow \circ}(t) x_{\circ \rightarrow i}(t)}\right]
$$

appears as a natural candidate for the limiting energy of any sequence of finite networks whose random weak limit is $\mathcal{L}$. Our second result is the validity of this cavity ansatz when $\mathcal{L}$ is concentrated on trees.

Theorem 4 (Asymptotical correctness of the cavity method) Let $\left(G_{n}\right)_{n \in \mathbb{N}}$ be a sequence of finite cavity-monotone networks admitting a random weak limit $\mathcal{L}$ which is concentrated on cavity-monotone trees. Assume that the local rank at a uniformly chosen vertex is uniformly integrable as $n \rightarrow \infty$. Then,

$$
\frac{U\left(G_{n} ; t\right)}{\left|V_{n}\right|} \underset{n \rightarrow \infty}{\longrightarrow} u(\mathcal{L} ; t) .
$$

If $\left|E_{n}\right|=O\left(\left|V_{n}\right|\right)$ and all the local measures take values in $\{0\} \cup K$ for a fixed compact $K \subseteq(0, \infty)$, then

$$
\begin{gathered}
\frac{1}{\left|V_{n}\right|} \log Z\left(G_{n} ; t\right) \quad \underset{n \rightarrow \infty}{\longrightarrow} \mathcal{L}\left[\log \mu_{\circ}(\emptyset)\right]+\int_{0}^{t} \frac{u(\mathcal{L} ; s)}{s} d s, \\
\frac{M\left(G_{n}\right)}{\left|V_{n}\right|} \quad \underset{n \rightarrow \infty}{\longrightarrow} m(\mathcal{L}):=\lim _{t \rightarrow \infty} \uparrow u(\mathcal{L} ; t) .
\end{gathered}
$$

Remark 2 (Large deviation principle) Integrating (20) immediately implies that

$$
\frac{1}{\left|V_{n}\right|} \log \frac{Z\left(G_{n} ; t\right)}{Z\left(G_{n} ; 1\right)} \underset{n \rightarrow \infty}{\longrightarrow} \int_{1}^{t} \frac{u(\mathcal{L} ; s)}{s} d s .
$$

It will later be checked that $t \mapsto u(\mathcal{L} ; t)$ is continuous on $\mathbb{R}_{+}$(Remark 3 ). Therefore, denoting by $\mathcal{F}_{n}$ a random spanning subgraph with the Gibbs-Boltzmann law $\mathbb{P}_{G_{n}}^{1}$, Gärtner-Ellis Theorem [1] guarantees that $\left|\mathcal{F}_{n}\right| /\left|V_{n}\right|$ obeys a large deviation principle with rate $\left|V_{n}\right|$ and good rate function $y \mapsto \int_{0}^{\infty}\left(y-u\left(\mathcal{L} ; e^{s}\right)\right)^{+} d s$. 


\subsection{Proof of the main result}

Lemma 4 (Tree approximation) Let $(G, \circ)$ be a finite rooted cavity-monotone network, and let $k \in \mathbb{N}$. If $[G, \circ]_{2 k+2}$ is a tree, then for every activity $t>0$,

$$
U_{\mu_{\circ}}\left(x_{i \rightarrow \circ}^{2 k}(t): i \in \partial \circ\right) \leq \mathbb{E}_{G}^{t}\left[\left|\mathcal{F} \cap E_{\circ}\right|\right] \leq U_{\mu_{\circ}}\left(x_{i \rightarrow \circ}^{2 k+1}(t): i \in \partial \circ\right)
$$

Proof. The proof makes use of a classical ingredient known as the spatial Markov property, which we first briefly recall. Let $G=(V, E)$ be a finite network and let $S$ be an induced subgraph. We let $\partial S$ denote the boundary of $S$, i.e. the set of edges having one end-point in $S$ and one in $S^{c}$. Any boundary condition $B \subseteq \partial S$ can be used to assign local measures to the vertices of $S$, namely $\mu_{i}^{B}(F):=\mu_{i}\left(F \cup\left(B \cap E_{i}\right)\right)$. Note that these local measures differ from the original ones only for vertices that are adjacent to the boundary. The resulting network is denoted by $S \mid B$. Now, a spanning subgraph $F \subseteq E$ is clearly the disjoint union of a spanning subgraph $F_{\text {int }}$ of $S$, a boundary condition $B \subseteq \partial S$ and a spanning subgraph $F_{\text {ext }}$ in $S^{c}$. The product form of $\mu_{G}$ immediately yields :

$$
\mathbb{P}_{G}^{t}(\mathcal{F}=F)=\mathbb{P}_{S \mid B}^{t}\left(\mathcal{F}=F_{\text {int }}\right) \mathbb{P}_{G}^{t}(\mathcal{F} \cap \partial S=B) \mathbb{P}_{S^{c} \mid B}^{t}\left(\mathcal{F}=F_{\text {ext }}\right)
$$

In other words, conditionally on the boundary $\mathcal{B}:=\mathcal{F} \cap \partial S$, the restrictions of $\mathcal{F}$ to $S$ and $S^{c}$ are independent with law $\mathbb{P}_{S \mid \mathcal{B}}^{t}$ and $\mathbb{P}_{S^{c} \mid \mathcal{B}}^{t}$, respectively. Applying this to the tree $S=[G, \circ]_{2 k+2}$,

$$
\begin{aligned}
\mathbb{E}_{G}^{t}\left[\left|\mathcal{F} \cap E_{\circ}\right|\right] & =\sum_{B \subseteq \partial S} \mathbb{P}_{G}^{t}(\mathcal{F} \cap \partial S=B) \mathbb{E}_{S \mid B}^{t}\left[\left|\mathcal{F} \cap E_{\circ}\right|\right] \\
& =\sum_{B \subseteq \partial S} \mathbb{P}_{G}^{t}(\mathcal{F} \cap \partial S=B) U_{\mu_{\circ}}\left(x_{i \rightarrow \circ}^{(B)}(t): i \in \partial \circ\right),
\end{aligned}
$$

where we have applied Lemma 3 to the tree $S \mid B$, writing $\mathbf{x}^{(B)}(t)$ for the unique solution to the cavity equation at activity $t$ thereon. But by monotony of the cavity operator, each $x_{i \rightarrow \circ}^{(B)}(t), i \in \partial \circ$ must satisfy $x_{i \rightarrow \circ}^{2 k}(t) \leq x_{i \rightarrow \circ}^{(B)}(t) \leq x_{i \rightarrow \circ}^{2 k+1}(t)$. Using the size-increasing property of $\mu_{\circ}$, we see that

$$
U_{\mu_{\circ}}\left(x_{i \rightarrow \circ}^{2 k}(t): i \in \partial \circ\right) \leq U_{\mu_{\circ}}\left(x_{i \rightarrow \circ}^{(B)}(t): i \in \partial \circ\right) \leq U_{\mu_{\circ}}\left(x_{i \rightarrow \circ}^{2 k+1}(t): i \in \partial \circ\right),
$$

and re-injecting this into the above equation finally yields the desired inequalities.

Let us now see how Lemma 4 implies the convergence (20). Let $\left(G_{n}\right)_{n \in \mathbb{N}}$ be a sequence of finite cavity-monotone networks admitting a random weak limit $\mathcal{L}$ which is concentrated on cavity-monotone trees. Denote by $\mathcal{L}_{n} \in \mathcal{P}\left(\mathcal{G}_{*}\right)$ the law under uniform rooting of $G_{n}$, so that $\mathcal{L}_{n} \Rightarrow \mathcal{L}$. We will use the short-hand $u_{k}(G, \circ)=U_{\mu_{\circ}}\left(x_{i \rightarrow \circ}^{k}(t): i \in \partial \circ\right)$, and $\chi_{k}(G, \circ)$ for the indicator function that $[G, \circ]_{2 k+2}$ is a tree. Lemma guarantees that for any finite cavity-monotone network $G$ and any vertex $\circ$,

$$
\chi_{k}(G, \circ) u_{2 k}(G, \circ) \leq \mathbb{E}_{G}^{t}\left[|\mathcal{F}| \cap E_{\circ}\right] \leq \chi_{k}(G, \circ) u_{2 k+1}(G, \circ)+\left(1-\chi_{k}(G, \circ)\right) \operatorname{rank}\left(\mu_{\circ}\right) .
$$

As functions of $(G, \circ)$, the left-hand side and right-hand side are continuous on $\mathcal{G}_{*}$, since they depend only on $[G, \circ]_{2 k+2}$. Moreover, both are dominated by $(G, \circ) \mapsto \operatorname{rank}\left(\mu_{\circ}\right)$ which is assumed to be uniformly integrable with respect to the sequence $\left(\mathcal{L}_{n}\right)_{n \in \mathbb{N}}$. Thus, their expectation under $\mathcal{L}_{n}$ tends to their expectation under $\mathcal{L}$ as $n \rightarrow \infty$. But $\chi_{k}$ is zero on the support of $\mathcal{L}$, so we are simply left with

$$
\frac{1}{2} \mathcal{L}\left[U_{\mu_{\circ}}\left(x_{i \rightarrow \circ}^{2 k}(t): i \in \partial \circ\right)\right] \leq \liminf _{n \rightarrow \infty} \frac{U\left(G_{n} ; t\right)}{\left|V_{n}\right|} \leq \limsup _{n \rightarrow \infty} \frac{U\left(G_{n} ; t\right)}{\left|V_{n}\right|} \leq \frac{1}{2} \mathcal{L}\left[U_{\mu_{\circ}}\left(x_{i \rightarrow \circ}^{2 k+1}(t): i \in \partial \circ\right)\right] .
$$


Since the random weak limit $\mathcal{L}$ is unimodular, Theorem 3 finally implies that both the lower and upper bounds tend to $\mathcal{L}\left[U_{\mu_{\circ}}\left(x_{i \rightarrow \circ}(t): i \in \partial \circ\right)\right]=u(\mathcal{L} ; t)$ as $k \rightarrow \infty$. Note that the requirement $\mathcal{L}\left[\operatorname{rank}\left(\mu_{\circ}\right)\right]<\infty$ in Theorem 3 is here automatically fullfilled, by the uniform integrability assumption.

It now remains to show (21) and (22). The identity (5) implies that for any activity $t>0$ and any finite network $G$ satisfying $\mu(\emptyset)>0$,

$$
\frac{1}{|V|} \log Z(G ; t)=\frac{1}{|V|} \sum_{\circ \in V} \log \mu_{\circ}(\emptyset)+\int_{0}^{t} \frac{U(G ; s)}{s|V|} d s .
$$

Now take $G=G_{n}$ and let $n \rightarrow \infty$ : the compactness assumption guarantees that $\log \mu_{\circ}(\emptyset)$ is bounded uniformly in $n$, so the first term converges to $\mathcal{L}\left[\log \mu_{\circ}(\emptyset)\right]$. As per the second one, it tends to $\int_{0}^{t} \frac{u(\mathcal{L} ; s)}{s} d s$ because of (20), provided the uniform domination holds in Lebesgue's dominated convergence Theorem. The latter fact is ensured by the first inequality in Lemma 5 below, combined with the compactness assumption and the fact that $\left|E_{n}\right|=O\left(\left|V_{n}\right|\right)$. The second inequality in Lemma 5 easily guarantees (22).

Lemma 5 (Uniform controls for the energy) Let $G$ be a finite cavity-monotone network. As a function of the activity $t$, the energy $U(G ; t)$ increases from 0 to $M(G)$. Furthermore, the rate of convergence to these two extrema can be precisely controlled:

$$
\begin{aligned}
& \forall t>0, \quad U(G ; t) \leq t \sum_{i j \in E} A\left(\mu_{i}\right) A\left(\mu_{j}\right) \\
& \forall t>1, \quad U(G ; t) \geq M(G)-\frac{1}{\log t}\left(|E| \log 2+\sum_{i \in V} \log A\left(\mu_{i}\right)\right) .
\end{aligned}
$$

where $A(\mu)=\frac{\max \mu}{\min \mu}$, with $\max \mu=\max \{\mu(F): F \in \operatorname{supp}(\mu)\}$ and $\min \mu=\min \{\mu(F): F \in \operatorname{supp}(\mu)\}$.

Proof of Lemma . That the energy increases with the activity is equivalent by (5) to the convexity of $\theta \mapsto \log Z\left(G ; e^{\theta}\right)$, a direct consequence of Hölder's inequality. This also implies that for any $t>1$

$$
U(G ; t) \log t \geq \log \frac{Z(G ; t)}{Z(G ; 1)} .
$$

Clearly $Z(G ; t) \geq t^{M(G)} \min \mu$ and $Z(G ; 1) \leq 2^{|E|} \max \mu$ and $A(\mu) \leq \prod_{i \in V} A\left(\mu_{i}\right)$, so (26) follows. Regarding (25), we have for any $t>0$

$$
\begin{aligned}
U(G ; t) & =\sum_{i j \in E} \mathbb{P}^{t}(i j \in \mathcal{F}) \\
& =t \sum_{i j \in E} \frac{\sum_{F \subseteq E \backslash i j} \mu(F \cup i j) t^{|F|}}{\sum_{F \subseteq E} \mu(F) t^{|F|}} \\
& \leq t \sum_{i j \in E} \max _{F \subseteq E \backslash i j, \mu(F)>0} \frac{\mu(F \cup i j)}{\mu(F)} \\
& \leq t \sum_{i j \in E} A\left(\mu_{i}\right) A\left(\mu_{j}\right),
\end{aligned}
$$

where the third line uses the standard inequality $\frac{a+b}{c+d} \leq \max \left(\frac{a}{c}, \frac{b}{d}\right)$ for any $a, b, c, d>0$, and the crucial fact that $\mu(F \cup i j)>0 \Longrightarrow \mu(F)>0$ (see Remark 1i). 


\section{The limit of infinite activity}

The goal of this final section is to describe the important quantity $m(\mathcal{L})$ introduced in Theorem $\mathbb{1}$ directly in terms of a certain local equation which we naturally call the cavity equation at infinite activity. This will then be used to establish the explicit formulae that have been mentioned in the introduction.

\subsection{The cavity equation at infinite activity}

Let $G$ be a cavity-monotone network. From Lemma 1, it follows that $(t, \mathbf{x}) \mapsto t \Gamma_{G}(t \mathbf{x})$ is increasing in $t$ and decreasing in $\mathbf{x}$. We may thus define a limiting cavity-operator by

$$
\bar{\Gamma}_{G}(\mathbf{x}):=\lim _{t \rightarrow \infty} \uparrow t \Gamma_{G}(t \mathbf{x}) .
$$

By monotony, $\bar{\Gamma}_{G}:[0, \infty)^{\vec{E}} \rightarrow(0, \infty]^{\vec{E}}$ is well-defined without any ambiguity regarding the order in which the limits $t \rightarrow \infty$ and $\mathbf{x} \rightarrow 0$ are taken. Note also that $\bar{\Gamma}_{G}$ can be composed with $\Gamma_{G}:(0, \infty]^{\vec{E}} \rightarrow[0, \infty)^{\vec{E}}$, yielding a two-step local update rule on $(0, \infty]^{\vec{E}}$ which will now play a crucial role.

Theorem 5 (The cavity equation at infinite activity) Let $G$ be a cavity-monotone network on which the cavity equation at activity $t$ admits a unique globally attractive fixed point $\mathbf{x}(t)$, for every $t>0$. Then,

$$
\overline{\mathbf{x}}:=\lim _{t \rightarrow \infty} \uparrow \mathbf{x}(t)
$$

exists in $(0, \infty]^{\vec{E}}$, and is the smallest solution to the so-called cavity equation at infinite activity on $G$ :

$$
\overline{\mathbf{x}}=\left(\bar{\Gamma}_{G} \circ \Gamma_{G}\right)(\overline{\mathbf{x}}) .
$$

In particular, for any unimodular probability measure $\mathcal{L}$ concentrated on cavity-monotone networks, and satisyfing $\mathcal{L}\left[\operatorname{rank}\left(\mu_{\circ}\right)\right]<\infty$, we have

$$
m(\mathcal{L})=\frac{1}{2} \mathcal{L}\left[U_{\mu_{\circ}}\left(\bar{x}_{i \rightarrow \circ}: i \in \partial \circ\right)\right]
$$

Proof of Theorem 5. By assumption $\mathbf{x}^{k}(t) \rightarrow \mathbf{x}(t)$ for any $t>0$, where $\mathbf{x}^{0} \equiv \mathbf{0}$ and for all $k \in \mathbb{N}$,

$$
\mathbf{x}^{k+1}(t)=t \Gamma_{G}\left(t \frac{\mathbf{x}^{k}(t)}{t}\right) \quad \text { or equivalently, } \quad \frac{\mathbf{x}^{k+1}(t)}{t}=\Gamma_{G}\left(\mathbf{x}^{k}(t)\right) .
$$

But $(t, \mathbf{x}) \mapsto t \Gamma_{G}(t \mathbf{x})$ is increasing in $t$ and decreasing in $\mathbf{x}$, so an immediate induction over $k$ shows that $t \mapsto t^{-1} \mathbf{x}^{k}(t)$ and $t \mapsto \mathbf{x}^{k}(t)$ are respectively non-increasing and non-decreasing, for every $k \in \mathbb{N}$. Thus, $t \mapsto \mathbf{x}(t)$ is non-decreasing, hence the existence of (27). The identity (28) is then obtained by passing to the limits in $(29)$. Finally, if $\mathbf{y} \in(0, \infty]^{\vec{E}}$ satisfies $\mathbf{y}=\left(\bar{\Gamma}_{G} \circ \Gamma_{G}\right)(\mathbf{y})$, then for every $k \in \mathbb{N}$ and $t>0$,

$$
\mathbf{x}^{2 k}(t) \leq \mathbf{y} .
$$

Indeed, the property is trivial when $k=0$, and is preserved from $k$ to $k+1$ because $t \Gamma_{G}(t \mathbf{w}) \leq \bar{\Gamma}_{G}(\mathbf{w})$ for any $\mathbf{w} \in[0, \infty)^{\vec{E}}$. Letting $k \rightarrow \infty$ and then $t \rightarrow \infty$ in (30) yields $\overline{\mathbf{x}} \leq \mathbf{y}$, as desired.

Remark 3 (Continuity with respect to the activity) Incidentally, we have just obtained that $t \mapsto$ $t^{-1} \mathbf{x}(t)$ and $t \mapsto \mathbf{x}(t)$ are respectively non-increasing and non-decreasing, so that

$$
0<s \leq t \Longrightarrow \frac{s}{t} \mathbf{x}(t) \leq \mathbf{x}(s) \leq \mathbf{x}(t)
$$

This guarantees the continuity of $t \mapsto \mathbf{x}(t)$, and hence that of $u(\mathcal{L} ; t)$, as promised in Remark . 


\subsection{Explicit resolution for $b$-matchings on GW trees}

Many classical sequences of diluted random graphs admit almost surely a particularly simple random weak limit $\mathcal{L}$, namely a unimodular Galton-Watson $(U G W)$ tree (see Example 1.1 in [3]). This random rooted tree is parametrized by a probability distribution $\pi \in \mathcal{P}(\mathbb{N})$ with finite mean, called its degree distribution. It is obtained by a Galton-Watson branching process where the root has offspring distribution $\pi$ and all other genitors have the size-biased offspring distribution $\widehat{\pi} \in \mathcal{P}(\mathbb{N})$ defined by

$$
\forall n \in \mathbb{N}, \widehat{\pi}_{n}=(n+1) \pi_{n+1} / \sum_{k} k \pi_{k} .
$$

Thanks to the markovian nature of the branching process, the cavity equation at infinite activity simplifies into a recursive distributional equation (RDE) (see [2] for a survey). Let us describe it and solve it in the case of $b$-matchings, where $b \geq 1$ is fixed. The local cavity and energy ratios are simply

$$
\Gamma\left(x_{1}, \ldots, x_{n}\right)=\frac{\sum_{I \subseteq[N]:|I| \leq b-1} \prod_{i \in I} x_{i}}{\sum_{I \subseteq[N]:|I| \leq b} \prod_{i \in I} x_{i}} \quad \text { and } \quad U\left(x_{1}, \ldots, x_{n}\right)=\frac{\sum_{I \subseteq[N]:|I| \leq b}|I| \prod_{i \in I} x_{i}}{\sum_{I \subseteq[N]:|I| \leq b} \prod_{i \in I} x_{i}} .
$$

In the infinite activity limit, the local cavity ratio becomes

$$
\bar{\Gamma}\left(x_{1}, \ldots, x_{n}\right)=\frac{\sum_{I \subseteq[N]:|I|=b-1} \prod_{i \in I} x_{i}}{\sum_{I \subseteq[N]:|I|=b} \prod_{i \in I} x_{i}},
$$

where all conventions regarding degenerate cases are obtained by taking the corresponding limits. Given $Q \in \mathcal{P}((0, \infty])$, we let $\Theta(Q) \in \mathcal{P}([0,1]))$ denote the law of $\Gamma\left(Y_{1}, \ldots, Y_{\widehat{N}}\right)$, where $\widehat{N}$ has law $\widehat{\pi}$ and $Y_{1}, Y_{2}, \ldots$ are i.i.d. with law $Q$, independent of $\widehat{N}$. Similarly, given $P \in \mathcal{P}([0,1])$, we let $\bar{\Theta}(P) \in \mathcal{P}((0, \infty])$ denote the law of $\bar{\Gamma}\left(X_{1}, \ldots, X_{\widehat{N}}\right)$, where $\widehat{N}$ has law $\widehat{\pi}$ and $X_{1}, X_{2}, \ldots$ are i.i.d. with law $P$, independent of $\widehat{N}$. Thanks to the markovian nature of the GW branching process, the law $Q \in \mathcal{P}((0, \infty])$ of a message sent towards the root in the configuration $\overline{\mathbf{x}}$ must satisfy the $\operatorname{RDE} Q=(\bar{\Theta} \circ \Theta)(Q)$. Equivalently, $P=\Theta(Q)$ must satisfy $P=(\Theta \circ \bar{\Theta})(P)$. More precisely, letting $M(P)$ denote the expectation of $\frac{1}{2} U\left(Y_{1}, \ldots, Y_{N}\right)$, where $N$ has law $\pi$ and $Y_{1}, Y_{2}, \ldots$ are i.i.d. with law $Q=\bar{\Theta}(P)$, independent of $N$, we may rephrase Theorem 5 as follows (distributions are here endowed with the usual stochastic order) :

Corollary $1 m(\mathcal{L})=M(P)$, where $P \in \mathcal{P}([0,1])$ is the smallest solution to the $R D E P=(\Theta \circ \bar{\Theta})(P)$.

The fixed points of $\Theta \circ \bar{\Theta}$ turn out to be in one-to-one correspondance with the historical minima of a certain function $H:[0,1] \rightarrow \mathbb{R}$ defined in terms of the degree generating function $\phi(s)=\sum_{k} \pi_{k} s^{k}$ :

$$
\begin{gathered}
H(s)=b-\frac{b}{2} g(s)-\frac{b}{2}(g \circ f)(s)+\frac{c}{2} f(s)(f \circ f)(s), \\
\text { with } \quad c=\phi^{\prime}(1), \quad f(s)=\frac{1}{c} \sum_{k=0}^{b-1} \frac{s^{k} \phi^{(k+1)}(1-s)}{k !} \quad \text { and } \quad g(s)=\sum_{k=0}^{b} \frac{t^{k} \phi^{(k)}(1-s)}{k !} .
\end{gathered}
$$

A historical minima of $H$ is a number $s \in[0,1]$ satisfying $H^{\prime}(s)=0$ and $H(t)>H(s)$ for all $t \in[0, s)$.

Theorem 6 (Resolution of the RDE) Let $s_{1}<\ldots<s_{r}$ denote the historical minima of the function $H$. Then, the distributional equation $P=(\Theta \circ \bar{\Theta})(P)$ admits exactly $r$ solutions, and they are stochastically ordered : $P_{1}<\ldots<P_{r}$. Moreover, for each $1 \leq i \leq r$, we have $M\left(P_{i}\right)=H\left(s_{i}\right)$.

In particular, $m(\mathcal{L})=\min H$, which is exactly the formula given in Theorem 1. Theorem 6 was established in 14] for the special case $b=1$, but the proof can easily be adapted to the general case. For the sake of completeness, we have included a general proof in the Appendix. 


\section{Appendix : resolution of the $\mathrm{RDE}$}

First observe that the mappings $\Theta, \bar{\Theta}$ and $M$ are all decreasing with respect to stochastic order, and continuous with respect to the topology of weak convergence. Note also that $c \widehat{f}^{\prime}(t) t=b f(t)$, so that $H^{\prime}(t)=c f^{\prime}(t)((f \circ f)(t)-t)$. Thus, $H^{\prime}(t)=0$ if and only if $(f \circ f)(t)=t$.

\section{Lemma 6 (Properties of $\Gamma, \bar{\Gamma}$ and $U$ )}

1. Let $\left(x_{1}, \ldots, x_{n}\right) \in[0,1]^{n}$. Then,

(a) $\bar{\Gamma}\left(x_{1}, \ldots, x_{n}\right)=\infty \Longleftrightarrow \sum_{i=1}^{n} \mathbf{1}_{\left\{x_{i}>0\right\}}<b$;

(b) Setting $y_{i}=\bar{\Gamma}\left(x_{k}: k \neq i\right)$, we have $\sum_{i=1}^{n} \frac{x_{i} y_{i}}{1+x_{i} y_{i}} \mathbf{1}_{\left\{y_{i}<\infty\right\}}=b \mathbf{1}_{\left\{\sum_{i=1}^{n} \mathbf{1}_{\left\{x_{i}>0\right\}}>b\right\}}$

2. Let $\left(y_{1}, \ldots, y_{n}\right) \in(0, \infty]^{n}$. Then,

(a) $\Gamma\left(y_{1}, \ldots, y_{n}\right)>0 \Longleftrightarrow \sum_{i=1}^{n} \mathbf{1}_{\left\{y_{i}=\infty\right\}}<b$;

(b) Setting $x_{i}^{\prime}=\Gamma\left(y_{k}: k \neq i\right)$, we have $\sum_{i=1}^{n} \frac{x_{i}^{\prime} y_{i}}{1+x_{i}^{\prime} y_{i}} \mathbf{1}_{\left\{x_{i}^{\prime}<\infty\right\}}=U\left(x_{1}^{\prime}, \ldots, x_{n}^{\prime}\right)-b \wedge \sum_{i=1}^{n} \mathbf{1}_{\left\{y_{i}=\infty\right\}}$.

Proof. Properties 1.a and 2.a are straightforward from the definition of $\bar{\Gamma}$ and $\Gamma$. Regarding property $1 . b$, set $K=\#\left\{i \in[n]: x_{i}>0\right\}$. If the sum is non-zero then there must be an $i$ satisfying both $y_{i}>0$ and $x_{i}<\infty$. By 1.a, this implies $K>b$. Conversely, if $K>b$ then $x_{i}<\infty$ for every $i \in[n]$. We have just shown

$$
\begin{aligned}
\sum_{i=1}^{n} \frac{x_{i} y_{i}}{1+x_{i} y_{i}} \mathbf{1}_{\left\{y_{i}<\infty\right\}} & =\mathbf{1}_{\{K>b\}} \sum_{i=1}^{n} \frac{x_{i} y_{i}}{1+x_{i} y_{i}} \\
& =\mathbf{1}_{\{K>b\}} \sum_{i=1}^{n} \frac{\sum_{|I|=b, I \ni i} \prod_{k \in I} x_{k}}{\sum_{|I|=b} \prod_{k \in I} x_{k}} \\
& =b \mathbf{1}_{\{K>b\}},
\end{aligned}
$$

where the second equality is obtained by replacing $y_{i}=\bar{\Gamma}\left(x_{k}: k \neq i\right)$ by its definition. For property $2 . b$, set $L=\#\left\{i \in[n]: y_{i}=\infty\right\}$. When $L=0,2 . b$ boils down to formula (11). The case $1 \leq L \leq b$ can then be obtained by successively setting each of the $L$ variables to $\infty$, which amounts to condition on the presence of the corresponding ground elements. For $L \geq b$, both sides of the equation are zero.

Lemma 7 Assume that $P \stackrel{\bar{\theta}}{\rightarrow} Q \stackrel{\theta}{\rightarrow} P^{\prime}$. Set $s=P\left(\{0\}^{c}\right), t=Q(\{\infty\})$ and $s^{\prime}=P^{\prime}\left(\{0\}^{c}\right)$. Then,

$$
\begin{aligned}
& \text { 1. } s \stackrel{f}{\rightarrow} t \stackrel{f}{\rightarrow} s^{\prime} ; \\
& \text { 2. } P^{\prime} \leq P \Longrightarrow M(P) \leq H(s) ; \\
& \text { 3. } P^{\prime} \geq P \Longrightarrow M(P) \geq H(s) .
\end{aligned}
$$

In particular, if $P=(\Theta \circ \bar{\Theta})(P)$ then $M(P)=H(s)$ and $H^{\prime}(s)=0$. 
Proof. In the whole proof, $N$ denotes a generic random integer with law $\pi, \widehat{N}$ a generic random integer with law $\widehat{\pi}, X, X_{1}, X_{2}, \ldots$ generic $(0, \infty]$-valued random variables with law $P, Y, Y_{1}, Y_{2}, \ldots$ generic $[0,1]$-valued random variables with law $Q$, and $X^{\prime}, X_{1}^{\prime}, X_{2}^{\prime}, \ldots$ generic $(0, \infty]$-valued random variables with law $P^{\prime}$. We use the convention that all variables appearing under the same expectation are independent. With these notations, properties 1.a and 2.a in Lemma 6 give

$$
\mathbb{P}(Y=\infty)=\mathbb{P}\left(\sum_{i=1}^{\widehat{N}} \mathbf{1}_{\left\{X_{i}>0\right\}}<b\right) \quad \text { and } \quad \mathbb{P}\left(X^{\prime}>0\right)=\mathbb{P}\left(\sum_{i=1}^{\widehat{N}} \mathbf{1}_{\left\{Y_{i}=\infty\right\}}<b\right),
$$

which, in view of the definition of $f$, yields exactly the first claim of the Lemma. Now, using property $1 . b$ and $2 . b$, we respectively obtain the two following identities :

$$
\begin{aligned}
\mathbb{E}\left[\frac{X Y}{1+X Y} \mathbf{1}_{\{Y<\infty\}}\right] & =\sum_{n \in \mathbb{N}} \widehat{\pi}(n) \mathbb{E}\left[\frac{X \bar{\Gamma}\left(X_{1}, \ldots, X_{n-1}\right)}{1+X \bar{\Gamma}\left(X_{1}, \ldots, X_{n-1}\right)} \mathbf{1}_{\left.\left\{\bar{\Gamma}\left(X_{1}, \ldots, X_{n-1}\right)<\infty\right\}\right]}\right] \\
& =\sum_{n \in \mathbb{N}} \pi(n) n \mathbb{E}\left[\frac{X \bar{\Gamma}\left(X_{1}, \ldots, X_{n-1}\right)}{1+X \bar{\Gamma}\left(X_{1}, \ldots, X_{n-1}\right)} \mathbf{1}_{\left.\left\{\bar{\Gamma}\left(X_{1}, \ldots, X_{n-1}\right)<\infty\right\}\right]}\right] \\
& =\sum_{n \in \mathbb{N}} \pi(n) \mathbb{E}\left[\sum_{i=1}^{n} \frac{X_{i} \bar{\Gamma}\left(X_{k}: k \neq i\right)}{1+X_{i} \bar{\Gamma}\left(X_{k}: k \neq i\right)} \mathbf{1}_{\left.\left\{\bar{\Gamma}\left(X_{k}: k \neq i\right)<\infty\right\}\right]}\right] \\
& =b \mathbb{P}\left(\sum_{i=1}^{N} \mathbf{1}_{\left\{X_{i}>0\right\}}>b\right) \\
& =b(1-g(s)) . \\
\mathbb{E}\left[\frac{X^{\prime} Y}{1+X^{\prime} Y} \mathbf{1}_{\{Y<\infty\}}\right] & =\sum_{n \in \mathbb{N}} \widehat{\pi}(n) \mathbb{E}\left[\frac{Y \Gamma\left(Y_{1}, \ldots, Y_{n-1}\right)}{1+Y \Gamma\left(Y_{1}, \ldots, Y_{n-1}\right)} \mathbf{1}_{\{Y<\infty\}}\right] \\
& =\sum_{n \in \mathbb{N}} \pi(n) n \mathbb{E}\left[\frac{Y \Gamma\left(Y_{1}, \ldots, Y_{n-1}\right)}{1+Y \Gamma\left(Y_{1}, \ldots, Y_{n-1}\right)} \mathbf{1}_{\{Y<\infty\}}\right] \\
& =\sum_{n \in \mathbb{N}} \pi(n) \mathbb{E}\left[\sum_{i=1}^{n} \frac{Y_{i} \Gamma\left(Y_{k}: k \neq i\right)}{1+Y_{i} \Gamma\left(Y_{k}: k \neq i\right)} \mathbf{1}_{\left\{Y_{i}<\infty\right\}}\right] \\
& =\mathbb{E}\left[U\left(Y_{1}, \ldots, Y_{N}\right)\right]-\mathbb{E}\left[b \wedge \sum_{i=1}^{N} \mathbf{1}_{\left\{Y_{i}=\infty\right\}}\right] \\
& =2 M(P)-b(1-g(s))-c s f(s) .
\end{aligned}
$$

Since the mapping $\frac{x y}{1+x y}$ is increasing in $x$, claims 2 and 3 follow.

Proof of Theorem G. Fix $s \in[0,1]$ satisfying $H^{\prime}(s)=0$, i.e. $(f \circ f)(s)=s$. Define $P_{0}^{s} \in \mathcal{P}([0,1])$ to be the Bernoulli distribution with parameter $s$, and set then iteratively

$$
P_{n+1}^{s}=(\Theta \circ \bar{\Theta})\left(P_{n}^{s}\right)
$$

for all $n \in \mathbb{N}$. By part 1 in Lemma $7, P_{1}^{s}$ is a distribution on $[0,1]$ satisfying $P_{1}\left(\{0\}^{c}\right)=s$. Since $P_{0}^{s}$ is the largest such distribution, we have $P_{1}^{s} \leq P_{0}^{s}$. But both $\Theta$ and $\bar{\Theta}$ are decreasing, so by immediate 
induction, the sequence $\left(P_{n}^{s}\right)_{n \in \mathbb{N}}$ is non-increasing. Thus, the limit $P^{s}=\lim _{n \rightarrow \infty} \downarrow P_{n}^{s}$ exists in $\mathcal{P}([0,1])$. Setting $s_{\infty}=P^{s}\left(\{0\}^{c}\right)$, we claim that

1. $P^{s}$ is a fixed point of $\Theta \circ \bar{\Theta}$;

2. $M\left(P^{s}\right)=H\left(s_{\infty}\right)$;

3. $s_{\infty} \leq s$;

4. $H\left(s_{\infty}\right) \leq H(s)$.

Part 1 follows from the continuity of $\Theta$ and $\bar{\Theta}$. Part 2 is guaranteed by Lemma 7 . Part 3 is a consequence of the fact that $P^{s} \leq P_{0}^{s}$. Finally, for each $n \in \mathbb{N}$, we have $P_{n}\left(\{0\}^{c}\right)=s$ and $P_{n}^{s} \geq P_{n+1}^{s}$, so Lemma 7 guarantees that $M\left(P_{n}^{s}\right) \leq H(s)$. Letting $n \rightarrow \infty$ yields exactly part 4 .

We are now in position to prove the equivalence between the historical minima of $H$ and the solutions to $(\Theta \circ \bar{\Theta})(P)=P$. If $s$ is a historical minimum, then parts 3 and 4 force $s_{\infty}=s$ so $P=P^{s}$ is a solution satisfying $M(P)=H(s)$, as desired. Conversely, we must show that any solution $P$ is in fact of the form $P^{s}$ for some historical minimum $s$. Set $s=P\left(\{0\}^{c}\right)$, which satisfies $H^{\prime}(s)=0$ by Lemma 7. We have $P \leq P_{n}^{s}$ for any $n \in \mathbb{N}$ : this holds for $n=0$ because $P_{0}^{s}$ is the largest element of $\mathcal{P}([0,1])$ such that $P_{0}\left(\{0\}^{c}\right)=s$, and it then inductively extends to all $n \in \mathbb{N}$ by monotony of $\Theta \circ \bar{\Theta}$. Letting $n \rightarrow \infty$, we obtain $P \leq P^{s}$; but by Lemma 7 we also have $M(P)=H(s)=M\left(P^{s}\right)$. Thus, $P=P^{s}(M$ is decreasing). Finally, if $t<s$ is any historical minimum then clearly $P_{0}^{t} \leq P_{0}^{s}$, which implies $P^{t} \leq P^{s}$. In fact the inequality is strict, because $P^{t}\left(\{0\}^{c}\right)=t<s=P^{s}\left(\{0\}^{c}\right)$. Applying the decreasing mapping $M$ yields $H(t)>H(s)$, which shows that $s$ is a historical minimum.

\section{References}

[1] D. Aldous. The $\zeta(2)$ limit in the random assignment problem. Random Structures Algorithms, 18(4):381-418, 2001.

[2] D. Aldous and A. Bandyopadhyay. A survey of max-type recursive distributional equations. Ann. Appl. Probab., 15(2):1047-1110, 2005.

[3] D. Aldous and R. Lyons. Processes on unimodular random networks. Electronic Journal of Probability, 12:1454-1508, 2007.

[4] D. Aldous and M. Steele. The objective method: probabilistic combinatorial optimization and local weak convergence. In Probability on discrete structures, volume 110 of Encyclopaedia Math. Sci., pages 1-72. Springer, Berlin, 2004.

[5] A. Bandyopadhyay and D. Gamarnik. Counting without sampling: asymptotics of the log-partition function for certain statistical physics models. Random Structures Algorithms, 33(4):452-479, 2008.

[6] M. Bayati, D. Gamarnik, D. Katz, C. Nair, and P. Tetali. Simple deterministic approximation algorithms for counting matchings. In Proceedings of the thirty-ninth annual ACM symposium on Theory of computing, page 127. ACM, 2007.

[7] M. Bayati and C. Nair. A rigorous proof of the cavity method for counting matchings. Proc. of the 44th Annual Allerton Conference on Communication, Control and Computing., 2006. 
[8] I. Benjamini and O. Schramm. Recurrence of distributional limits of finite planar graphs. Electron. J. Probab., 6:no. 23, 13 pp. (electronic), 2001.

[9] I. Benjamini, O. Schramm, and A. Shapira. Every minor-closed property of sparse graphs is testable. In STOC, pages 393-402, 2008.

[10] J. Berg. On the absence of phase transition in the monomer-dimer model. Technical report, Amsterdam, The Netherlands, 1998.

[11] P. Billingsley. Convergence of probability measures. Wiley Series in Probability and Statistics: Probability and Statistics. John Wiley \& Sons Inc., New York, second edition, 1999. A WileyInterscience Publication.

[12] T. Bohman and A. Frieze. Karp-Sipser on random graphs with a fixed degree sequence. 2009.

[13] C. Bordenave, M. Lelarge, and J. Salez. Maximum matchings in diluted graphs: the cavity method at positive temperature. in preparation.

[14] C. Bordenave, M. Lelarge, and J. Salez. The rank of diluted random graphs. to appear in Annals of Probability.

[15] C. Borgs, J. Chayes, J. Kahn, and L. Lovász. Left and right convergence of graphs with bounded degree. Arxiv preprint arXiv:1002.0115, 2010.

[16] A. Dembo and A. Montanari. Ising models on locally tree-like graphs. Ann. Appl. Probab., 20(2):565592,2010 .

[17] A. Dembo and O. Zeitouni. Large deviations techniques and applications, volume 38 of Stochastic Modelling and Applied Probability. Springer-Verlag, Berlin, 2010. Corrected reprint of the second (1998) edition.

[18] G. Elek. On limits of finite graphs. Combinatorica, 27(4):503-507, 2007.

[19] D. Gamarnik. Linear phase transition in random linear constraint satisfaction problems. Probab. Theory Related Fields, 129(3):410-440, 2004.

[20] D. Gamarnik, T. Nowicki, and G. Swirszcz. Maximum weight independent sets and matchings in sparse random graphs. Exact results using the local weak convergence method. Random Structures Algorithms, 28(1):76-106, 2006.

[21] O. Heilmann and E. Lieb. Theory of monomer-dimer systems. Comm. Math. Phys., 25:190-232, 1972.

[22] J. Kahn and M. Neiman. Negative correlation and log-concavity. Random Structures Algorithms, $37(3): 367-388,2010$.

[23] R. Karp and M. Sipser. Maximum matchings in sparse random graphs. Proc. of the Twenty-second Annual Symposium on Foundations of Computer Science, IEEE:364-375, 1981.

[24] L. Lovász and M. Plummer. Matching theory. AMS Chelsea Publishing, Providence, RI, 2009. Corrected reprint of the 1986 original [MR0859549].

[25] R. Lyons. Asymptotic enumeration of spanning trees. Combin. Probab. Comput., 14(4):491-522, 2005. 
[26] M. Mézard, G. Parisi, and M. A. Virasoro. Spin glass theory and beyond, volume 9 of World Scientific Lecture Notes in Physics. World Scientific Publishing Co. Inc., Teaneck, NJ, 1987.

[27] R. Pemantle. Towards a theory of negative dependence. J. Math. Phys., 41(3):1371-1390, 2000. Probabilistic techniques in equilibrium and nonequilibrium statistical physics.

[28] T. Richardson and R. Urbanke. The capacity of low-density parity-check codes under messagepassing decoding. IEEE Trans. Inform. Theory, 47(2):599-618, 2001.

[29] D. Ruelle. Counting unbranched subgraphs. J. Algebraic Combin., 9(2):157-160, 1999.

[30] A. Schrijver. Combinatorial optimization. Polyhedra and efficiency. Vol.A, volume 24 of Algorithms and Combinatorics. Springer-Verlag, Berlin, 2003. Paths, flows, matchings, Chapters 1-38.

[31] M. Talagrand. Spin glasses: a challenge for mathematicians, volume 46 of Results in Mathematics and Related Areas. 3rd Series. A Series of Modern Surveys in Mathematics. Springer-Verlag, Berlin, 2003. Cavity and mean field models.

[32] L. Valiant. The complexity of computing the permanent. Theoret. Comput. Sci., 8(2):189-201, 1979.

[33] D. Wagner. Negatively correlated random variables and Mason's conjecture for independent sets in matroids. Ann. Comb., 12(2):211-239, 2008.

[34] D. Wagner. Weighted enumeration of spanning subgraphs with degree constraints. J. Combin. Theory Ser. B, 99(2):347-357, 2009. 